\title{
Conductance of Carbon Nanotube Junctions in Magnetic Fields
}

\author{
Hajime Matsumura and Tsuneya ANDO \\ Institute for Solid State Physics, University of Tokyo \\ 5-1-5 Kashiwanoha, Kashiwa, Chiba 277-8581
}

\begin{abstract}
The conductance of $\mathrm{CN}$ junction in the presence of a magnetic field perpendicular to the tube axis is calculated in an effective-mass approximation. By making modification of the development, envelope functions in the junction region are obtained analytically in the presence of a magnetic field when $\varepsilon=0$. The conductance exhibits little dependence on the magnetic field, contrary to the results of tight-binding calculations.
\end{abstract}

\section{$\S 1$. Introduction}

Recent synthetic techniques have made it possible to obtain various carbon structures based on fullerenes. A carbon nanotube $(\mathrm{CN})$ was first produced by means of the arc-discharge evaporation method. ${ }^{1)} \mathrm{A} \mathrm{CN}$ is composed of coaxially rolled graphite sheets. A single-shell CN can be either a metal or a semiconductor depending on the circumferential length and the helical fashion. The purpose of this paper is to calculate the conductance of a $\mathrm{CN}$ junction in magnetic fields.

Various energy band calculations have been performed. ${ }^{2-12)}$ It has been found that these characteristic properties are reproduced well in a $\boldsymbol{k} \cdot \boldsymbol{p}$ method, ${ }^{13,14)}$ which is effective in the study of effects of external fields such as a magnetic and electric field. In fact, the effective-mass scheme has been used successfully in the study of wide varieties of electronic properties of $\mathrm{CN}$, such as magnetic properties ${ }^{15}$ ) including the Aharonov-Bohm effect on the band gap, ${ }^{13)}$ optical absorption spectra, ${ }^{16,17)}$ exciton effects, ${ }^{18)}$ lattice instabilities in the absence ${ }^{19)}$ and presence of a magnetic field, ${ }^{20,21)}$ and magnetic properties of ensembles of nanotubes. ${ }^{22)}$ Thus, $\boldsymbol{k} \cdot \boldsymbol{p}$ method is quite useful when we study the low-energy electronic properties of CN's.

Topological effects on the electronic states of CN system are interesting. Carbon nanotubes with a pentagon-heptagon pair were observed. ${ }^{23)}$ A junction which connects tubes with different diameters has been observed in the transmission electron microscope. Some theoretical calculations on $\mathrm{CN}$ junctions within a tightbinding model were reported, which imply that the conductance of junctions exhibits a universal power-law dependence on the ratio of two tubes connected through the junction. ${ }^{24-26)}$ This behavior has been understood in the $\boldsymbol{k} \cdot \boldsymbol{p}$ scheme based on characteristic boundary conditions for envelope functions around a topological defect such as pentagonal and heptagonal rings. ${ }^{27)}$ Effects of a magnetic field were studied also and intriguing dependence on the magnetic-field direction was predicted. ${ }^{28,29)}$

In this paper we shall discuss electronic states and transport of CN junction in the $\boldsymbol{k} \cdot \boldsymbol{p}$ scheme in magnetic fields. In $\S 2$ a kind of scale transformation is introduced for the purpose of relating the wave function in a magnetic field to that in its absence. In $\S 3$, the method of the wave-function matching is discussed. The numerical results are given in $\S 4$. A summary and conclusions are given in $\S 5$.

\section{$\S 2$. Junctions in Magnetic Fields}

\subsection{Effective-Mass Description}

In a two-dimensional graphite a unit cell contains two carbon atoms denoted as A and B and the conduction and valence bands consisting of $\pi$ orbitals have a linear dispersion and cross each other at $\mathrm{K}$ and $\mathrm{K}$ ' points of the hexagonal first Brillouin zone. ${ }^{30}$ ) States in the vicinity of the Fermi level chosen at $\varepsilon=0$ are described in an effective-mass scheme by four-component envelope wave functions

$$
\boldsymbol{F}(\boldsymbol{r})=\left(\begin{array}{c}
\boldsymbol{F}^{K}(\boldsymbol{r}) \\
\boldsymbol{F}^{K^{\prime}}(\boldsymbol{r})
\end{array}\right)
$$

with

$$
\boldsymbol{F}^{K}(\boldsymbol{r})=\left(\begin{array}{c}
\boldsymbol{F}_{A}^{K}(\boldsymbol{r}) \\
\boldsymbol{F}_{B}^{K}(\boldsymbol{r})
\end{array}\right), \quad \boldsymbol{F}^{K^{\prime}}(\boldsymbol{r})=\left(\begin{array}{c}
\boldsymbol{F}_{A}^{K^{\prime}}(\boldsymbol{r}) \\
\boldsymbol{F}_{B}^{K^{\prime}}(\boldsymbol{r})
\end{array}\right)
$$

where $F_{A}^{K}$ and $F_{B}^{K}$ describe a slowly-varying part of the wave function at $\mathrm{A}$ and $\mathrm{B}$ sites, respectively, for states in the vicinity of the $\mathrm{K}$ point, and $F_{A}^{K^{\prime}}$ and $F_{B}^{K^{\prime}}$ describe that for states in the vicinity of the $K^{\prime}$ point. ${ }^{31,13)}$ They satisfy the Schrödinger equation

$$
\mathcal{H} \boldsymbol{F}(\boldsymbol{r})=\varepsilon \boldsymbol{F}(\boldsymbol{r})
$$

with

$$
\begin{gathered}
\mathcal{H}=\left(\begin{array}{cc}
\mathcal{H}_{K} & 0 \\
0 & \mathcal{H}_{K^{\prime}}
\end{array}\right) \\
\mathcal{H}_{K}=\left(\begin{array}{cc}
0 & \gamma\left(\hat{k}_{x}-\mathrm{i} \hat{k}_{y}\right) \\
\gamma\left(\hat{k}_{x}+\mathrm{i} \hat{k}_{y}\right) & 0
\end{array}\right),
\end{gathered}
$$

and

$$
\mathcal{H}_{K^{\prime}}=\left(\begin{array}{cc}
0 & \gamma\left(\hat{k}_{x}+\mathrm{i} \hat{k}_{y}\right) \\
\gamma\left(\hat{k}_{x}-\mathrm{i} \hat{k}_{y}\right) & 0
\end{array}\right) .
$$

In the presence of a magnetic field, $\hat{k}_{x}=(\partial / \mathrm{i} \partial x)+(e / c) A_{x}$ and $\hat{k}_{y}=(\partial / \mathrm{i} \partial y)+(e / c) A_{y}$, where $\boldsymbol{A}=\left(A_{x}, A_{y}\right)$ is a vector potential.

\subsection{Junctions}

The structure of a junction system is shown in Fig. 1. In a previous study, ${ }^{27)}$ we assumed the structure to be closer to Fig. 1 (a), but apparently the conductance is expected to be same for the structure shown in Fig. 1 (b). In fact, the topological structure determined by boundary conditions along the circumference direction 
and around the five- and seven-membered rings is equivalent and therefore Fig. 1 (b) is considered to be the most symmetric and simplest model describing essential ingredients of topological effects. Further, numerical results presented in the following justify this expectation at least in the absence of a magnetic field. In the following, therefore, we shall exclusively consider a junction given in Fig. 1 (b) in a magnetic field applied perpendicular to the axis.

We separate the junction into three regions, a thicker nanotube with circumference $L_{5}$, a junction region where the circumference $L$ varies along the axis direction chosen in the $y$ direction, and a thinner nanotube with circumference $L_{7}$. There is a five-membered ring (whose position is denoted as $\boldsymbol{R}_{5}$ ) at the boundary of the thicker nanotube and the junction region and a seven-membered $\operatorname{ring}\left(\boldsymbol{R}_{7}\right)$ at the boundary of the junction region and the thinner nanotube. The junction is developed onto two dimension as schematically illustrated in Fig. 2.

\subsection{Scale Transformation} $(r, \theta)$

In the junction region, we use polar coordinates

$$
x=r \cos \theta, \quad y=r \sin \theta .
$$

As shown in Fig. 3, the position in the junction region can be specified also by $\zeta$, which takes $\zeta=+\pi$ at $\theta=-\pi / 3$ and $\zeta=-\pi$ at $\theta=-2 \pi / 3$, i.e.

$$
x=\frac{L \zeta}{2 \pi} .
$$

The relation between $\theta$ and $\zeta$ is given by

$$
\zeta=6 \theta+3 \pi \text {. }
$$

The boundary with the thick nanotube with circumference $L_{5}$ is given by $r=r_{5}$ with $r_{5}=3 L_{5} / \pi$ and that with the thin nanotube with $L_{7}$ is given by $r=r_{7}$ with $r_{7}=3 L_{7} / \pi$

Let $H^{\prime}$ be the magnetic field perpendicular to the cylinder surface at $\zeta=\zeta_{0}$. Then, the effective magnetic field is given by $H^{\prime} \cos \left(\zeta-\zeta_{0}\right)=-H^{\prime} \cos \left(6 \theta-\zeta_{0}\right)$ and the corresponding vector potential is given by

$$
A_{r}=-\frac{H^{\prime} r}{6} \sin \left(6 \theta-\zeta_{0}\right)
$$

We have

$$
\begin{aligned}
& \hat{k}_{x}-\mathrm{i} \hat{k}_{y}=\mathrm{e}^{-\mathrm{i} \theta}\left[\frac{\partial}{\partial r}-\frac{\mathrm{i}}{r} \frac{\partial}{\partial \theta}-\frac{\mathrm{i} r}{6 l^{\prime 2}} \sin \left(6 \theta-\zeta_{0}\right)\right], \\
& \hat{k}_{x}+\mathrm{i} \hat{k}_{y}=\mathrm{e}^{+\mathrm{i} \theta}\left[\frac{\partial}{\partial r}+\frac{\mathrm{i}}{r} \frac{\partial}{\partial \theta}-\frac{\mathrm{i} r}{6 l^{\prime 2}} \sin \left(6 \theta-\zeta_{0}\right)\right],
\end{aligned}
$$

where $l^{\prime}=\sqrt{c \hbar / e H^{\prime}}$.

In the followings, we shall obtain the function $\varphi_{ \pm}(r, \theta)$ satisfying the equation:

$$
\begin{aligned}
& \left(\frac{\partial}{\partial r}-\frac{\mathrm{i}}{r} \frac{\partial}{\partial \theta}\right) \varphi_{-}(r, \theta)-\frac{\mathrm{i} r}{6 l^{\prime 2}} \sin \left(6 \theta-\zeta_{0}\right)=0, \\
& \left(\frac{\partial}{\partial r}+\frac{\mathrm{i}}{r} \frac{\partial}{\partial \theta}\right) \varphi_{+}(r, \theta)+\frac{\mathrm{i} r}{6 l^{\prime 2}} \sin \left(6 \theta-\zeta_{0}\right)=0,
\end{aligned}
$$

and $\varphi_{ \pm}(\zeta+2 \pi)=\varphi_{ \pm}(\zeta)$. To solve this, we put

$$
\varphi_{ \pm}(r, \theta)=\frac{r^{2}}{l^{\prime 2}} \mathrm{e}^{ \pm 2 \mathrm{i} \theta} \varphi_{ \pm}^{\prime}(\theta) .
$$

Then, we have

$$
\begin{aligned}
& \frac{\partial \varphi_{-}^{\prime}(\theta)}{\partial \theta}=-\frac{1}{6} \mathrm{e}^{+2 \mathrm{i} \theta} \sin \left(6 \theta-\zeta_{0}\right), \\
& \frac{\partial \varphi_{+}^{\prime}(\theta)}{\partial \theta}=+\frac{1}{6} \mathrm{e}^{-2 \mathrm{i} \theta} \sin \left(6 \theta-\zeta_{0}\right) .
\end{aligned}
$$

These can be solved as

$$
\begin{aligned}
& \varphi_{-}^{\prime}(\theta)=C_{-}+\frac{1}{96}\left[\mathrm{e}^{+6 \mathrm{i} \theta-\mathrm{i} \zeta_{0}}+2 \mathrm{e}^{-6 \mathrm{i} \theta+\mathrm{i} \zeta_{0}}\right] \mathrm{e}^{+2 \mathrm{i} \theta}, \\
& \varphi_{+}^{\prime}(\theta)=C_{+}-\frac{1}{96}\left[\mathrm{e}^{-6 \mathrm{i} \theta+\mathrm{i} \zeta_{0}}+2 \mathrm{e}^{+6 \theta-\mathrm{i} \zeta_{0}}\right] \mathrm{e}^{-2 \mathrm{i} \theta}
\end{aligned}
$$

with $C_{ \pm}$being constants. The condition $\varphi_{ \pm}(\zeta+2 \pi)=$ $\varphi_{ \pm}(\zeta)$ leads to $C_{ \pm}=0$. Thus, we have

$$
\begin{aligned}
& \varphi_{-}(r, \theta)=+\frac{1}{32}\left(\frac{r}{l^{\prime}}\right)^{2}\left[\cos \left(\zeta-\zeta_{0}\right)-\frac{\mathrm{i}}{3} \sin \left(\zeta-\zeta_{0}\right)\right], \\
& \varphi_{+}(r, \theta)=-\frac{1}{32}\left(\frac{r}{l^{\prime}}\right)^{2}\left[\cos \left(\zeta-\zeta_{0}\right)+\frac{\mathrm{i}}{3} \sin \left(\zeta-\zeta_{0}\right)\right] .
\end{aligned}
$$

As shown in Fig. 4, the circumference for a given $r$ is given by $L=(\pi / 3) r$ and therefore the radius is given by $L / 2 \pi=(1 / 6) r$. This gives the apex angle $\xi$ satisfying $\sin \xi=1 / 6$ and $\cos \xi=\sqrt{35} / 6$. Consequently, we have $H^{\prime}=H \cos \xi=(\sqrt{35} / 6) H$ and

$$
\begin{aligned}
& \varphi_{-}(r, \theta)=+\frac{3 \sqrt{35}}{16}\left(\frac{L}{2 \pi l}\right)^{2}\left[\cos \left(\zeta-\zeta_{0}\right)-\frac{\mathrm{i}}{3} \sin \left(\zeta-\zeta_{0}\right)\right], \\
& \varphi_{+}(r, \theta)=-\frac{3 \sqrt{35}}{16}\left(\frac{L}{2 \pi l}\right)^{2}\left[\cos \left(\zeta-\zeta_{0}\right)+\frac{\mathrm{i}}{3} \sin \left(\zeta-\zeta_{0}\right)\right],
\end{aligned}
$$

where $l=\sqrt{c \hbar / e H}$.

Let $\mathcal{H}_{K}^{0}$ be the Hamiltonian in the absence of a magnetic field for the $\mathrm{K}$ point. Then, we have the relation

$$
P^{-1} \mathcal{H}_{K} P=\mathcal{H}_{K}^{0},
$$

where $P$ is defined by

$$
P=\left(\begin{array}{cc}
\exp \left[\varphi_{+}\left(L, \zeta-\zeta_{0}\right)\right] & 0 \\
0 & \exp \left[\varphi_{-}\left(L, \zeta-\zeta_{0}\right)\right]
\end{array}\right),
$$

with

$$
\begin{aligned}
& \varphi_{-}(L, \zeta)=+\frac{3 \sqrt{35}}{16}\left(\frac{L}{2 \pi l}\right)^{2}\left(\cos \zeta-\frac{\mathrm{i}}{3} \sin \zeta\right), \\
& \varphi_{+}(L, \zeta)=-\frac{3 \sqrt{35}}{16}\left(\frac{L}{2 \pi l}\right)^{2}\left(\cos \zeta+\frac{\mathrm{i}}{3} \sin \zeta\right) .
\end{aligned}
$$

Since we have the envelope functions for $\varepsilon=0$ in the absence of a magnetic field, we obtain those in a magnetic field through this nonunitary transformation:

$$
\boldsymbol{F}_{H=0}^{K} \rightarrow \boldsymbol{F}_{H \neq 0}^{K}=P \boldsymbol{F}_{H=0}^{K} .
$$

For the K' point, we have

$$
P^{\prime-1} \mathcal{H}_{K^{\prime}} P^{\prime}=\mathcal{H}_{K^{\prime}}^{0}
$$


where $P^{\prime}$ is defined by

$$
P^{\prime}=\left(\begin{array}{cc}
\exp \left[\varphi_{-}\left(L, \zeta-\zeta_{0}\right)\right] & 0 \\
0 & \exp \left[\varphi_{+}\left(L, \zeta-\zeta_{0}\right)\right]
\end{array}\right) .
$$

We obtain the envelope functions for $\varepsilon=0$ in the presence of a magnetic field from those in the absence of magnetic field as

$$
\boldsymbol{F}_{H=0}^{K^{\prime}} \rightarrow \boldsymbol{F}_{H \neq 0}^{K^{\prime}}=P^{\prime} \boldsymbol{F}_{H=0}^{K^{\prime}} .
$$

Envelope functions in the junction region localize in the direction of the magnetic field $\left(\zeta=\zeta_{0}\right.$ and $\left.\zeta \pm \pi\right)$ in sufficiently strong fields .

Similarly, wave functions in the tube region are given from zero-field solutions through the nonunitary transformation, ${ }^{32)}$ using matrix $P_{0}$ and $P_{0}^{\prime}$ defined as

$$
P_{0}=\left(\begin{array}{cc}
\exp \left[\varphi_{+}^{0}\left(L, x, \zeta_{0}\right)\right] & 0 \\
0 & \exp \left[\varphi_{-}^{0}\left(L, x, \zeta_{0}\right)\right]
\end{array}\right),
$$

and

$$
P_{0}^{\prime}=\left(\begin{array}{cc}
\exp \left[\varphi_{-}^{0}\left(L, x, \zeta_{0}\right)\right] & 0 \\
0 & \exp \left[\varphi_{+}^{0}\left(L, x, \zeta_{0}\right)\right]
\end{array}\right),
$$

with

$$
\varphi_{ \pm}^{0}\left(L, x, \zeta_{0}\right)=\exp \left[ \pm\left(\frac{L}{2 \pi l}\right)^{2} \cos \left(\frac{2 \pi x}{L}-\zeta_{0}\right)\right] .
$$

These transformations show that the wave function is localized around $\zeta_{0}$ or $\zeta_{0} \pm \pi$ in a strong magnetic field in both tube and junction region. A comparison between $\varphi_{ \pm}$given by eq. (2.20) and $\varphi_{ \pm}^{0}$ given by eq. (2.27) shows that the wave function is more strongly localized in the junction region than in the tube region with same circumference because $3 \sqrt{35} / 16=1.10 \ldots>$ 1. This seems somewhat strange because the effective magnetic field perpendicular to the surface is weaker in the junction region, but is presumably related to the presence of the phase term in $\varphi_{ \pm}$proportional to $\sin (\zeta-$ $\left.\zeta_{0}\right)$.

\subsection{Boundary Conditions}

For metallic tubes, the wave functions satisfy usual periodic boundary conditions and are given by a set of plane waves. In the vicinity of the Fermi energy, we have four conducting modes in tube regions: right- (' $\mathrm{K}+$ ' and ' $\mathrm{K}$ '+') and left-going (' $\mathrm{K}-$ ' and ' $\mathrm{K}$ '-') waves associated with $\mathrm{K}$ and $\mathrm{K}$ ' points in the absence of a magnetic field. They are given by eqs. (3.41) and (3.42) of ref. 27. For $\mathrm{K} \pm$ states in the absence of a magnetic field, for example, we have at $\varepsilon=0$

$$
\boldsymbol{F}_{H=0}^{K \pm}=\frac{1}{\sqrt{2 L A}}\left(\begin{array}{c}
1 \\
\pm \mathrm{i}
\end{array}\right)
$$

where $A$ is the length of $\mathrm{CN}$. The corresponding wave function in magnetic fields is given by

$$
\boldsymbol{F}_{0}^{K \pm}=\frac{1}{\sqrt{I_{0}(2 \alpha)}} P^{0} \boldsymbol{F}_{H=0}^{K \pm},
$$

where $I_{0}(t)$ is the modified Bessel function of the first kind defined as

$$
I_{0}(t)=\int_{0}^{\pi} \frac{\mathrm{d} \theta}{\pi} \exp (t \cos \theta)
$$

and $\alpha=(L / 2 \pi l)^{2}$. The group velocity is given by

$$
v=\frac{\gamma}{\hbar I_{0}(2 \alpha)}
$$

and the density of states

$$
D(0)=\frac{I_{0}(2 \alpha)}{\pi \gamma}
$$

We should note that

$$
I_{0}(2 \alpha) \approx \begin{cases}1+\alpha^{2}+\cdots & (\alpha \ll 1) \\ \mathrm{e}^{2 \alpha} / \sqrt{4 \pi \alpha} & (\alpha \gg 1)\end{cases}
$$

This means that the group velocity for states at $\varepsilon=0$ decreases and consequently the density of states increases exponentially with the increase of the magnetic field in high-field regime. Further, the wave function normalized in such a way that it carries a unit flux is given by $P^{0} \boldsymbol{F}_{H=0}^{K \pm}$ which contains the magnetic field only in the scale factor $P$.

In addition to these modes, there are evanescent modes decaying in the left direction $\left(\boldsymbol{F}_{n}^{K-}\right.$ and $\boldsymbol{F}_{n}^{K^{\prime}-}$ with integer $n(n \neq 0)$ given by eqs. (4.1) and (4.2) of ref. 27 in the absence of a magnetic field) and those decaying in the right direction $\left(\boldsymbol{F}_{n}^{K+}\right.$ and $\boldsymbol{F}_{n}^{K^{\prime}+}$ given by eqs (4.3) and (4.4) of ref. 27 in the absence of a magnetic field).

Boundary conditions take a different form in the junction region, where any point on the development moves onto the corresponding point after making a rotation by $\pi / 3$ around $\boldsymbol{R}$ as shown in Fig. 2 . We have

$$
\begin{aligned}
& \boldsymbol{F}\left[R_{\pi / 3} \boldsymbol{r}\right]=T_{\pi / 3} \boldsymbol{F}(\boldsymbol{r}), \\
& T_{\pi / 3}=\left(\begin{array}{cccc}
0 & 0 & 0 & \omega^{1 / 2} \\
0 & 0 & -\omega^{-1 / 2} & 0 \\
0 & -\omega^{-1 / 2} & 0 & 0 \\
\omega^{1 / 2} & 0 & 0 & 0
\end{array}\right),
\end{aligned}
$$

where $\omega=\exp (2 \pi i / 3)$ and $R_{\pi / 3}$ describes a $\pi / 3$ rotation around $\boldsymbol{R}$. Because of the boundary conditions, states near the $\mathrm{K}$ and $\mathrm{K}^{\prime}$ point mix together in the junction region. The wave functions at $\varepsilon=0$ in the absence of a magnetic field are given by $\boldsymbol{F}_{m}^{J A}$ and $\boldsymbol{F}_{m}^{J B}$ with integer $m$ (eqs. (4.8) and (4.9) of ref. 27). As has been shown in the previous section, the wave functions in magnetic fields can be obtained from those in the absence of a field just by multiplying the scale factor (also a phase factor in the junction region).

\section{$\S 3$. Connection of Wave Functions}

In a previous paper, ${ }^{27}$ ) the wave functions at the boundary were expanded into a Fourier series and their coefficients were matched directly for the connection at the boundaries of the tube and junction regions. Although this Fourier-mode matching worked well in the absence of a magnetic field, it has some uncertainty on the convergence criteria.

In this paper we shall minimize the quantity $\Delta$ defined below, which is the sum of the differences of wave 
functions squared and integrated on each interface.

$$
\begin{aligned}
\Delta & =\Delta_{5}+\Delta_{7}, \\
\Delta_{5} & =\frac{1}{2 \pi} \int d \zeta\left|\boldsymbol{F}_{5}^{J}(\zeta)-\boldsymbol{F}_{5}^{T}(\zeta)\right|^{2}, \\
\Delta_{7} & =\frac{1}{2 \pi} \int d \zeta\left|\boldsymbol{F}_{7}^{J}(\zeta)-\boldsymbol{F}_{7}^{T}(\zeta)\right|^{2},
\end{aligned}
$$

where $\Delta_{5}$ is the integrated difference on the thicker tube side and $\Delta_{7}$ is that on the thinner tube side. The wave functions $\boldsymbol{F}_{5}^{J}$ and $\boldsymbol{F}_{7}^{J}$ are those in the junction region at the boundary $r=r_{5}$ with the thick tube and with the thin tube $r=r_{7}$, defined as

$$
\begin{aligned}
& \boldsymbol{F}_{5}^{J}(\zeta)=\sum_{m} \sum_{\alpha=A, B} C_{m \alpha}^{J} \boldsymbol{F}_{m}^{J \alpha}\left(r_{5}, \theta\right), \\
& \boldsymbol{F}_{7}^{J}(\zeta)=\sum_{m} \sum_{\alpha=A, B} C_{m \alpha}^{J} \boldsymbol{F}_{m}^{J \alpha}\left(r_{7}, \theta\right),
\end{aligned}
$$

with $\alpha=A$ and $B$, where $C_{m \alpha}^{J}$ is the coefficient to be determined, and $\boldsymbol{F}_{m}^{J A}(r, \theta)$ and $\boldsymbol{F}_{m}^{J B}(r, \theta)$ are the wave functions in the junction region expressed by the polar coordinates. The wave functions $\boldsymbol{F}_{5}^{T}$ and $\boldsymbol{F}_{7}^{T}$ are those in the tube regions at the boundaries, defined as

$$
\begin{aligned}
& \boldsymbol{F}_{5}^{T}(\zeta)=\sum_{n} \sum_{\beta=K-, K^{\prime}-, K+, K^{\prime}+} C_{n \beta}^{L} \boldsymbol{F}_{n}^{\beta}\left(L_{5}, \zeta\right), \\
& \boldsymbol{F}_{7}^{T}(\zeta)=\sum_{n} \sum_{\beta=K+, K^{\prime}+, K-, K^{\prime}-}^{R} C_{n \beta}^{R} \boldsymbol{F}_{n}^{\beta}\left(L_{7}, \zeta\right),
\end{aligned}
$$

where $C_{n \beta}^{L}$ and $C_{n \beta}^{R}$ are coefficients to be determined, and $\boldsymbol{F}_{n}^{\beta}(L, \zeta)$ are the wave functions in a tube with circumference $L$.

A direct evaluation of eq. (3.1) is likely to cause numerical difficulty. To see this, consider the wave functions around the five membered ring. In the absence of a magnetic field, as has been shown in ref. 27, the wave functions are proportional to $r^{(3 m-4) / 5}$ with $r$ being the distance from the center of the defect and $m$ being non-negative integers. For $m=0$, in particular, the line integral of the squared amplitude of wave functions along the path passing the defect center diverges at $r=0$ but the divergence should actually be cut off around $r \sim r_{c}$ where $r_{c}$ stands for a distance of the order of the lattice constant. Without such a proper cutoff the connection conditions may be dominated by contributions in the vicinity of the singularity due to the five-membered ring. The same is applicable to the seven-membered ring.

In order to avoid such problems, we expand every eigenfunction into Fourier series beforehand:

$$
\begin{aligned}
& \boldsymbol{F}_{m}^{J \alpha}\left(L_{5}, \zeta\right)=\frac{1}{\sqrt{L_{5}}} \sum_{\nu} \mathrm{e}^{\mathrm{i} \nu \zeta} \boldsymbol{f}_{m \alpha}^{J L}(\nu), \\
& \boldsymbol{F}_{m}^{J \alpha}\left(L_{7}, \zeta\right)=\frac{1}{\sqrt{L_{7}}} \sum_{\nu} \mathrm{e}^{\mathrm{i} \nu \zeta} \boldsymbol{f}_{m \alpha}^{J R}(\nu),
\end{aligned}
$$

and

$$
\begin{aligned}
& \boldsymbol{F}_{n}^{\beta}\left(L_{5}, \zeta\right)=\frac{1}{\sqrt{L_{5}}} \sum_{\nu} \mathrm{e}^{\mathrm{i} \nu \zeta} \boldsymbol{f}_{n \beta}^{L}(\nu), \\
& \boldsymbol{F}_{n}^{\beta}\left(L_{7}, \zeta\right)=\frac{1}{\sqrt{L_{7}}} \sum_{\nu} \mathrm{e}^{\mathrm{i} \nu \zeta} \boldsymbol{f}_{n \beta}^{R}(\nu),
\end{aligned}
$$

where $\nu$ is an integer. Then $\Delta$ is expressed as follows:

$$
\begin{aligned}
\Delta= & \sum_{\nu} \sum_{\alpha=A, B \beta=K-, K^{\prime}-, K+, K^{\prime}+} \sum \\
& \times\left(f_{c}\left(2 \pi \nu / L_{5}\right)^{2}\left|\boldsymbol{f}_{m \alpha}^{J L}(\nu) C_{m \alpha}^{J}-\boldsymbol{f}_{n \beta}^{L}(\nu) C_{n \beta}^{L}\right|^{2}\right. \\
& \left.+f_{c}\left(2 \pi \nu / L_{7}\right)^{2}\left|\boldsymbol{f}_{m \alpha}^{J R}(\nu) C_{m \alpha}^{J}-\boldsymbol{f}_{n \beta}^{R}(\nu) C_{n \beta}^{R}\right|^{2}\right),
\end{aligned}
$$

where we have introduced a cutoff function $f_{c}(\kappa)$ given by

$$
f_{c}(\kappa)=\frac{k_{c}{ }^{2}}{k_{c}^{2}+2 \kappa^{2}} .
$$

The cutoff $k_{c}$ should be chosen such that $2 \pi / k_{c} \sim a$, where $a$ is the lattice constant.

\section{$\S 4$. Numerical Results}

Before presenting the results in magnetic fields, we should examine how much this modification from Fig. 1 (a) to (b) affects the conductance in the absence of a magnetic field. Figure 5 shows the results of calculations for two structures in the case $\varepsilon=0$ and $H=0$. Both calculations are made using Fourier-mode matching. The calculated conductance agrees with each other within the numerical accuracy. Further, we compare the zero-field results obtained through two methods, the Fourier-mode matching and the present least-square method in Fig. 6. Differences between the results are negligibly small.

Figures 7, 8, and 9 show the results for $L_{7} / L_{5}=0.3$, 0.5 , and 0.8 with $\zeta_{0}=0$ and $2 \pi / k_{c} a=1$. The modes used are $-N_{\mathrm{mx}} \leq n \leq N_{\mathrm{mx}}$ in the tube region and $-2 N_{\mathrm{mx}}-1 \leq$ $m \leq 2 N_{\mathrm{mx}}$ in the junction region. ${ }^{27)}$ The circumference has been chosen as $L / a=10 \sqrt{3}$. With increasing number of modes involved in the calculation, total squared amplitude of scattering coefficients converges to unity (unitarity condition), while the magnetic-field dependence of the transmission probability becomes smaller and smaller. For the largest number of bases, the sum of the transmission and reflection probabilities becomes approximately unity in the field range $(L / 2 \pi l)^{2} \leq 2$, where the conductance remains independent of the magnetic field. In the highest field $(L / 2 \pi l)^{2} \gtrsim 4$, the convergence may be still unsatisfactory and the unitarity condition is slightly violated. However, it is safe to conclude that the conductance of the junction system is essentially independent of the magnetic field.

In the absence of a magnetic field, the transmission is possible only between same valleys, i.e., $t_{K K^{\prime}}=t_{K^{\prime} K}=$ 0 , and the reflection is between different valleys, i.e., $r_{K K}=r_{K^{\prime} K^{\prime}}=0$, for symmetric junctions shown in Fig. 1. This is a result of the mirror symmetry with respect to the plane containing the five- and seven-membered ring and the center of two cylinders. Although the results will not be shown explicitly, the same results hold in magnetic fields within the numerical accuracy.

These results are quite different from those obtained in tight-binding calculations. ${ }^{28}$ ) In fact, the tightbinding results have shown that the conductance for $\zeta_{0}=0$ decreases monotonically with the field when $0.5 \lesssim L_{7} / L_{5} \lesssim 1$ and exhibits a more complicated behavior when $L_{7} / L_{5} \lesssim 0.5$. Further, the transmission between 
different valleys and the reflection between same valleys become nonzero in contrast to the effective-mass result. Actually, the conductance in a tight-binding model is determined by $H \cos \zeta_{0}$, i.e., the field-component in the direction of the five- and seven-member rings and therefore, there is no field dependence for $\zeta_{0}= \pm \pi / 2$ in particular. Although not shown explicitly, the $\boldsymbol{k} \cdot \boldsymbol{p}$ result is independent of $\zeta_{0}$ within a numerical accuracy.

There can be various reasons for such discrepancy. In high magnetic fields, the wave function is localized and has a large amplitude in a narrow region and the density of states becomes exponentially large. ${ }^{15,32)}$ As a result even a weak scattering potential which does not cause any appreciable amount of scattering in the absence of a field may play a significant role. In the $\boldsymbol{k} \cdot \boldsymbol{p}$ scheme the presence of a five- and seven-member ring has been included only in the form of the boundary conditions for the envelop functions. When the discreteness of the lattice is considered, such a topological defect may effectively give rise to a weak and short-range potential. ${ }^{33)}$ In this case, such a potential causes the behavior of the conductance determined by $H \cos \zeta_{0}$ as in the case of short-range scatterers, ${ }^{34,35)}$ and the conductance varies as a function of the magnetic field in a complicated way depending on the difference in the number of impurities at $\mathrm{A}$ and $\mathrm{B}$ sites.

The change of the structure from Figs. 1 (a) to (b) may also be responsible to some of the discrepancy. In the absence of a magnetic field, the system does not have relevant length scales except $L_{5}$ and $L_{7}$ at $\varepsilon=0$ and therefore this change does not cause any significant effect. In magnetic fields, however, the magnetic length $l$ comes in and therefore the conductance is likely to be affected though slightly. The system given in Fig. 1 (b) is considered as most symmetric and simplest model describing essential ingredients of topological effects caused by the presence of five- and seven-membered rings.

The discrepancy might also come from the special characteristics of Weyl's equation at zero energy $\varepsilon=0$. In a numerical tight-binding calculation, the energy cannot be chosen exactly at zero, while the present analytic result is applicable only for zero energy. There may be a singularity at $\varepsilon=0$ and the result at $\varepsilon=0$ may be different from that at $\varepsilon \neq 0 .{ }^{36)}$ In order to clarify the origin of strange magnetic-field effects, we have to make extensive numerical calculations in both tight-binding model and $\boldsymbol{k} \cdot \boldsymbol{p}$ scheme. This problem is left for a future study.

The wave function in the presence of a magnetic field can be obtained from that in its absence simply by multiplying a scale factor in the tube regions and a scale and phase factor in the junction region as has been discussed in $\S 2$. The scale factors are almost same between the junction and tube regions. If a slight difference in the scale factor and the phase factor in the junction region are neglected completely, the condition for the connection of the wave functions becomes independent of the magnetic field. Further, the wave function with unit flux in the tube region contains the magnetic field only in the scale factor. Then the resulting conductance remains independent of a magnetic field. The numerical results presented above seem to show that effects of the phase factor are absorbed mostly by the inclusion of many evanescent modes in the tube regions.

\section{$\S 5$. Summary}

The conductance of $\mathrm{CN}$ junctions with magnetic fields applied perpendicularly to the tube axis has been calculated in the $\boldsymbol{k} \cdot \boldsymbol{p}$ scheme. For $\varepsilon=0$ wave functions both in tube and junction region have been obtained analytically from those in the absence of a magnetic field through a nonunitary scale transformation. The conductance of CN junctions has been calculated by connecting these wave functions at the interfaces between tube and junction region. Connection has been performed by variational method with a cutoff function with respect to the coefficients of eigenfunctions, which minimize the squared amplitudes of the difference between wave functions in both regions. The calculated conductance is independent of a magnetic field, and it is concluded that topological effects, which appear as a special boundary condition in the junction region, give little dependence of the conductance on magnetic fields. This result is contrary to that of tight-binding simulation. There might be several reasons for this discrepancy, which need to be investigated in the future.

\section{Acknowledgments}

This work was supported in part by Grants-in-Aid for Scientific Research and for Priority Area, Fullerene Network, from Ministry of Education, Science and Culture. One of us (H.M.) acknowledges the support of research fellowship from the Japan Society for the Promotion of Science for Young Scientists. Numerical calculations were performed in part using the facilities of the Supercomputer Center, Institute for Solid State Physics, University of Tokyo.

\section{References}

1) S. Iijima: Nature (London) 354 (1991) 56.

2) N. Hamada, S. Sawada and A. Oshiyama: Phys. Rev. Lett. 68 (1992) 1579.

3) J. W. Mintmire, B. I. Dunlap and C. T. White: Phys. Rev. Lett. 68 (1992) 631.

4) R. Saito, M. Fujita, G. Dresselhaus and M. S. Dresselhaus: Phys. Rev. B 46 (1992) 1804.

5) R. Saito, M. Fujita, G. Dresselhaus and M. S. Dresselhaus: Appl. Phys. Lett. 60 (1992) 2204.

6) M. S. Dresselhaus, G. Dresselhaus and R. Saito: Phys. Rev. B 45 (1992) 6234.

7) M. S. Dresselhaus, G. Dresselhaus, R. Saito and P. C. Eklund: Elementary Excitations in Solids, ed. J. L. Birman, C. Sebenne and R. F. Wallis (Elsevier Science Publishers B. V., Amsterdam, 1992) p. 387.

8) R. A. Jishi, M. S. Dresselhaus and G. Dresselhaus: Phys. Rev. B 47 (1993) 16671.

9) K. Tanaka, K. Okahara, M. Okada and T. Yamabe: Chem. Phys. Lett. 191 (1992) 469.

10) Y. D. Gao and W. C. Herndon: Mol. Phys. 77 (1992) 585.

11) D. H. Robertson, D. W. Brenner and J. W. Mintmire: Phys. Rev. B 45 (1992) 12592.

12) C. T. White, D. C. Robertson and J. W. Mintmire: 
Phys. Rev. B 47 (1993) 5485.

13) H. Ajiki and T. Ando: J. Phys. Soc. Jpn. 62 (1993) 1255 .

14) H. Ajiki and T. Ando: J. Phys. Soc. Jpn. 65 (1996) 505.

15) H. Ajiki and T. Ando: J. Phys. Soc. Jpn. 62 (1993) 2470. [Errata, J. Phys. Soc. Jpn. 63 (1994) 4267.]

16) H. Ajiki and T. Ando: Physica B 201 (1994) 349.

17) H. Ajiki and T. Ando: J. Phys. Soc. Jpn. 64 (1995) 4382 .

18) T. Ando: J. Phys. Soc. Jpn. 66 (1997) 1066.

19) N.A. Viet, H. Ajiki and T. Ando: J. Phys. Soc. Jpn. 63 (1994) 3036.

20) H. Ajiki and T. Ando, in The Physics of Semiconductors, edited by D.J. Lockwood (World Scientific, Singapore, 1995), p. 2061.

21) H. Ajiki and T. Ando: J. Phys. Soc. Jpn. 65 (1996) 2976.

22) H. Ajiki and T. Ando: Jpn. J. Appl. Phys. Suppl. 34-1 (1995) 107.

23) S. Iijima, T. Ichihashi and Y. Ando: Nature (London) 356 (1992) 776.

24) R. Tamura and M. Tsukada: Solid State Commun. 101 (1997) 601.

25) R. Tamura and M. Tsukada: Phys. Rev. B 55 (1997) 4991.

26) R. Tamura and M. Tsukada: Z. Phys. D 40 (1997) 432

27) H. Matsumura and T. Ando: J. Phys. Soc. Jpn. 67 (1998) 3542.

28) T. Nakanishi and T. Ando: J. Phys. Soc. Jpn. 66 (1997) 2973.

29) T. Nakanishi and T. Ando: Physica B 249-251 (1998) 136.

30) P. R. Wallace: Phys. Rev. 71 (1947) 622.

31) J. C. Slonczewski and P. R. Weiss: Phys. Rev. 109 (1958) 272.

32) T. Ando and T. Seri: J. Phys. Soc. Jpn. 66 (1997) 3558 .

33) R. Tamura and M. Tsukada: Phys. Rev. B 61 (2000) 8548.

34) M. Igami, T. Nakanishi and T. Ando: J. Phys. Soc. Jpn. 68 (1999) 716.

35) M. Igami, T. Nakanishi and T. Ando: J. Phys. Soc. Jpn. 70 (2001) 481.

36) H. Matsumura and T. Ando, J. Phys. Soc. Jpn. (submitted for publication).

\section{Figure Captions}

Fig. 1 A schematic illustration of a junction of a nanotube with circumference $L_{5}$ and another with $L_{7}$ sandwiched by a five-member ring $\boldsymbol{R}_{5}$ and a seven-member ring $\boldsymbol{R}_{7}$. The actual structure (a) is deformed into (b) in which both thicker and thinner tubes have a common axis and the whole structure is symmetric around the axis except for the presence of five- and seven-member rings. The structure (b) has the same topology as (a) and therefore is expected to give essentially the same conductance.

Fig. 2 A development of the junction system onto a two-dimensional plane. The polar coordinates $(r, \theta)$ are used in the junction region for which $-2 \pi / 3 \leq$ $\theta \leq-\pi / 3$ and $(3 / \pi) L_{7} \leq r \leq(3 / \pi) L_{5}$.

Fig. 3 The definition of the coordinate $\zeta$ on a circular circumference. The magnetic-field direction is denoted by $\zeta_{0}$.

Fig. 4 A schematic illustration of the junction region in which the apex angle $\xi$ is defined.

Fig. 5 Conductance for $\varepsilon=0$ in the absence of magnetic fields calculated for two kinds of developments. "Original" stands for the junction shown in Fig. 1(a) and "Conic" means the one shown in Fig. 1(b). The calculation method is Fourier-mode matching, with error limit $\delta=10^{-6}$.

Fig. 6 Conductance for $\varepsilon=0$ in the absence of magnetic fields calculated by two methods. "FMM" stands for the Fourier-mode matching and "LSM" means the least square method. For the latter, the modes $-N_{\mathrm{mx}} \leq n \leq N_{\mathrm{mx}}$ with $N_{\mathrm{mx}}=18$ are included in tube region.

Fig. 7 Calculated sum of the transmission and reflection probabilities (unitarity check) and conductance for $L_{7} / L_{5}=0.3$. With increasing number of modes involved in the calculation, total squared amplitude of scattering coefficients converges to unity as shown in (a) and the magnetic field dependence of the conductance becomes smaller as shown in (b).

Fig. 8 Calculated sum of the transmission and reflection probabilities (unitarity check) and conductance for $L_{7} / L_{5}=0.5$.

Fig. 9 Calculated sum of the transmission and reflection probabilities (unitarity check) and conductance for $L_{7} / L_{5}=0.8$. 


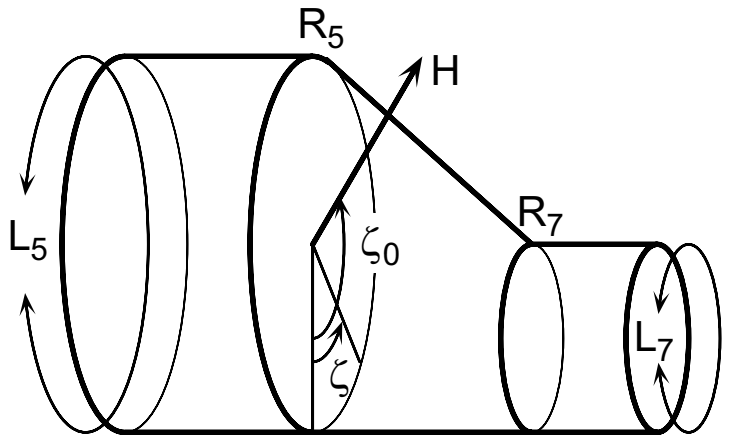

(a)

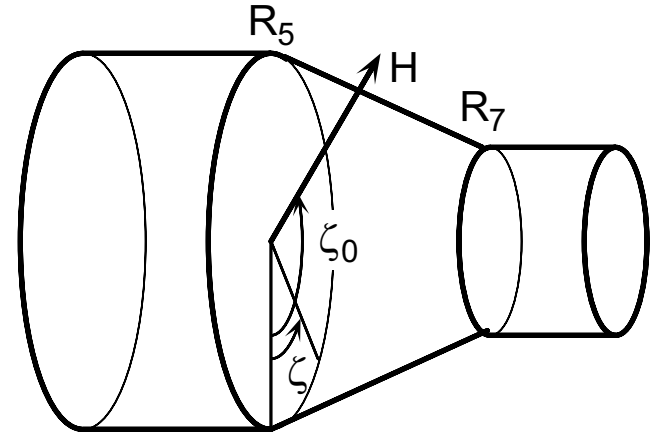

(b)

Fig. 1

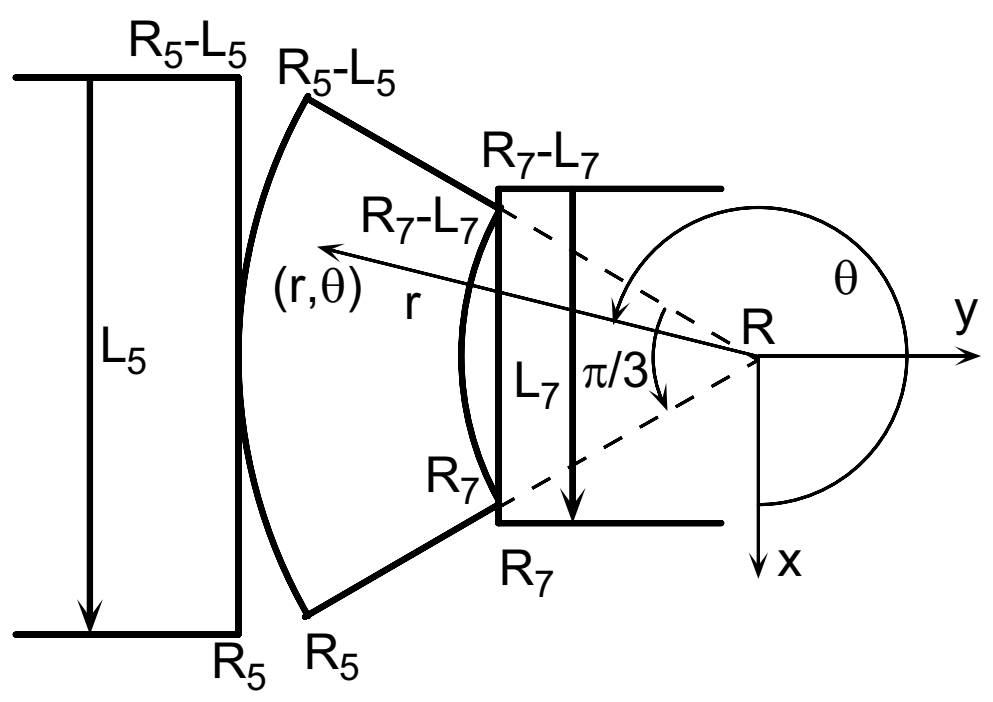

Fig. 2

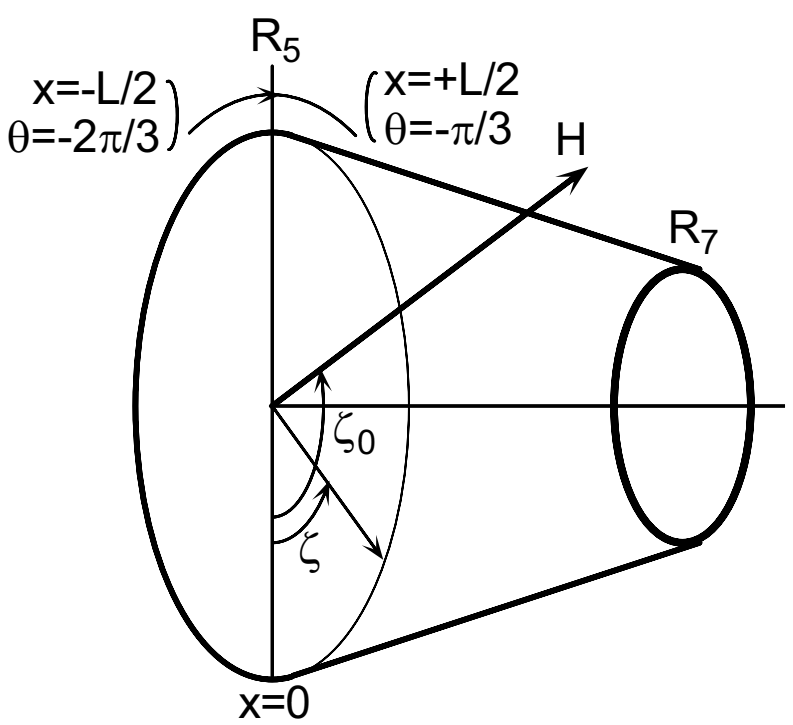

Fig. 3

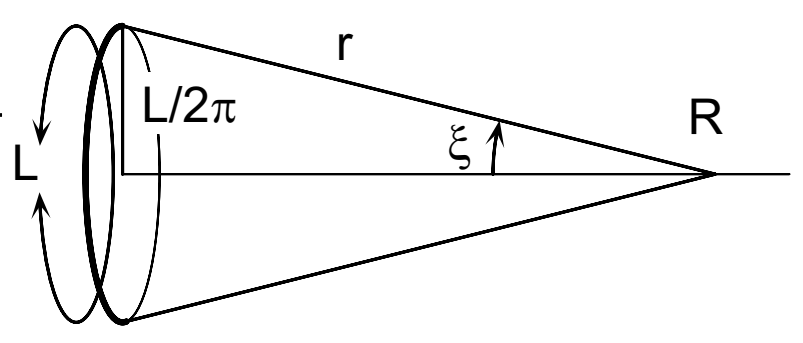

Fig. 4 


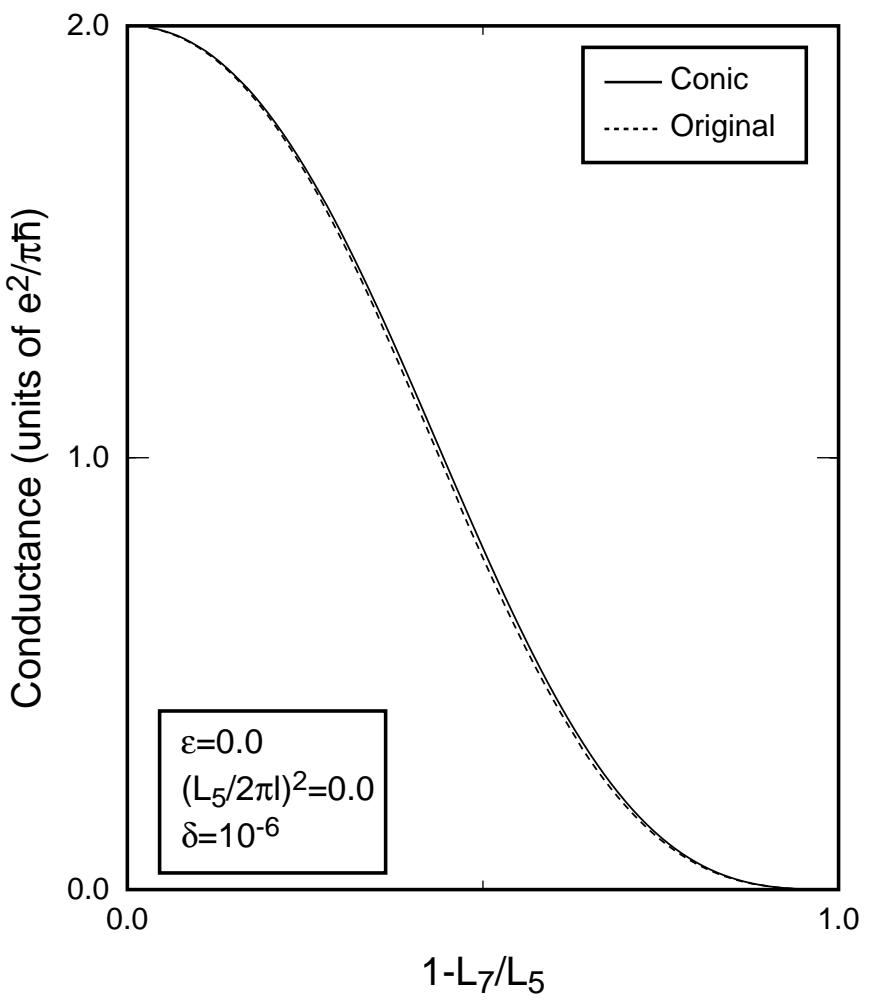

Fig. 5

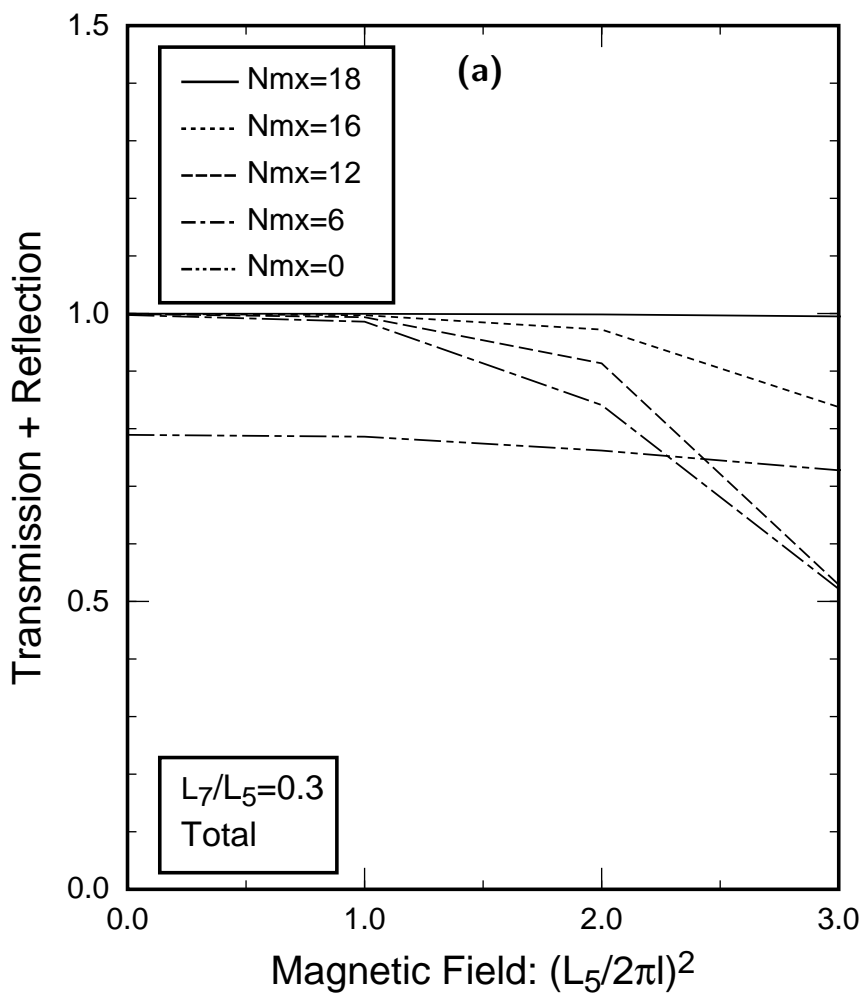

Fig. 7 (a)

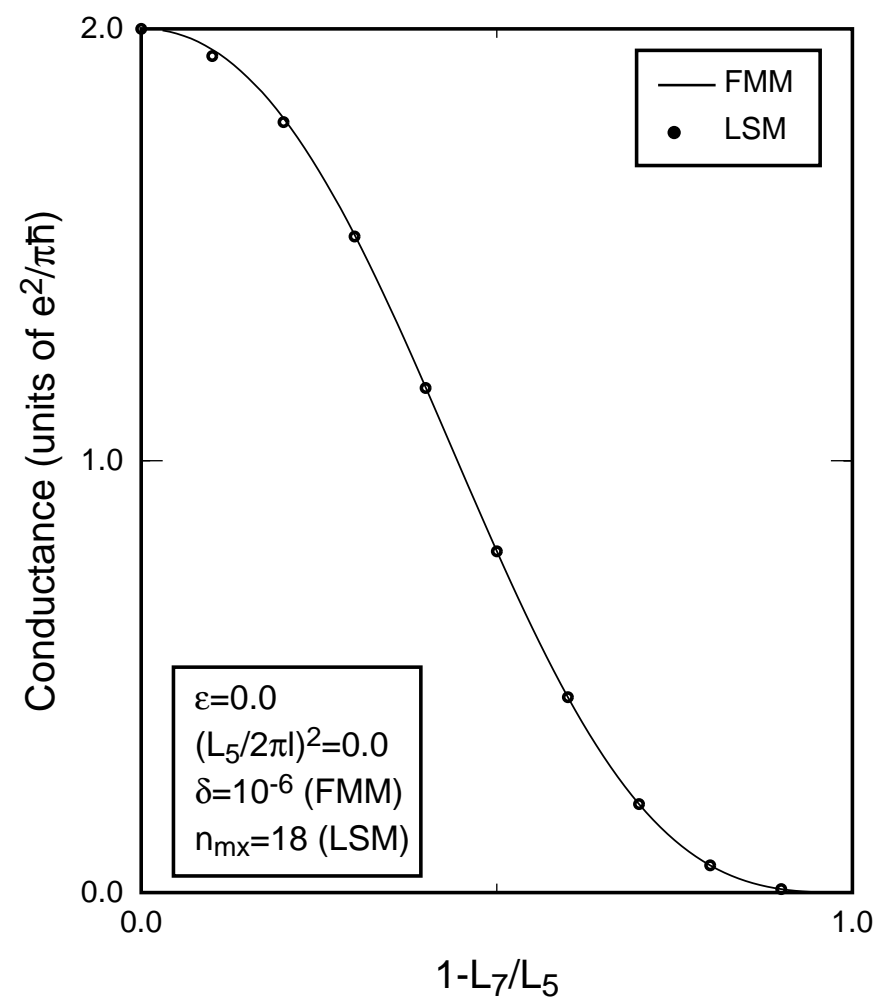

Fig. 6

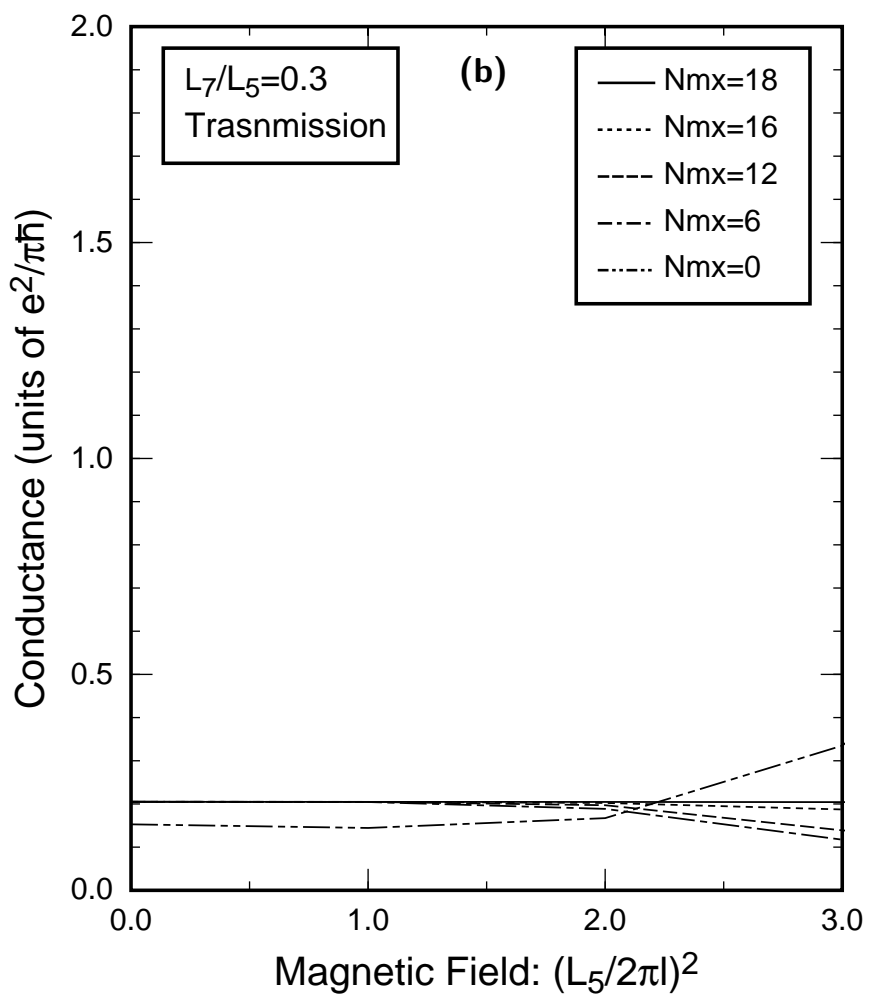

Fig. 7(b) 


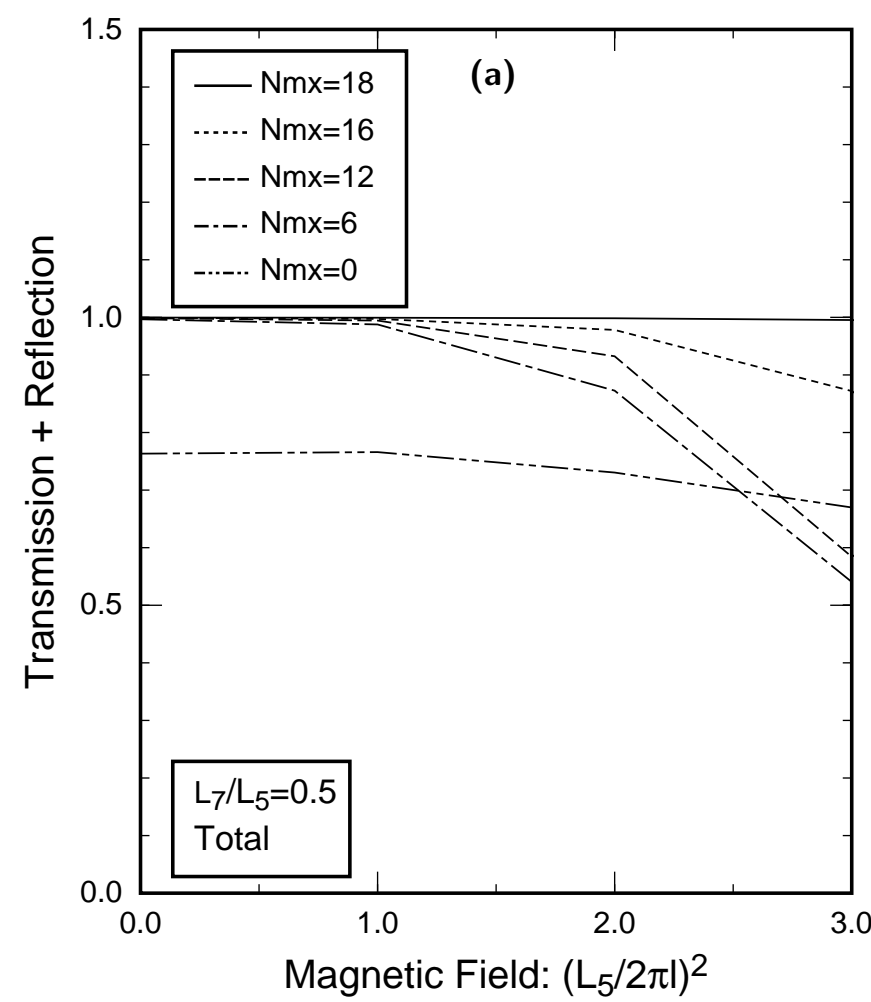

Fig. 8(a)

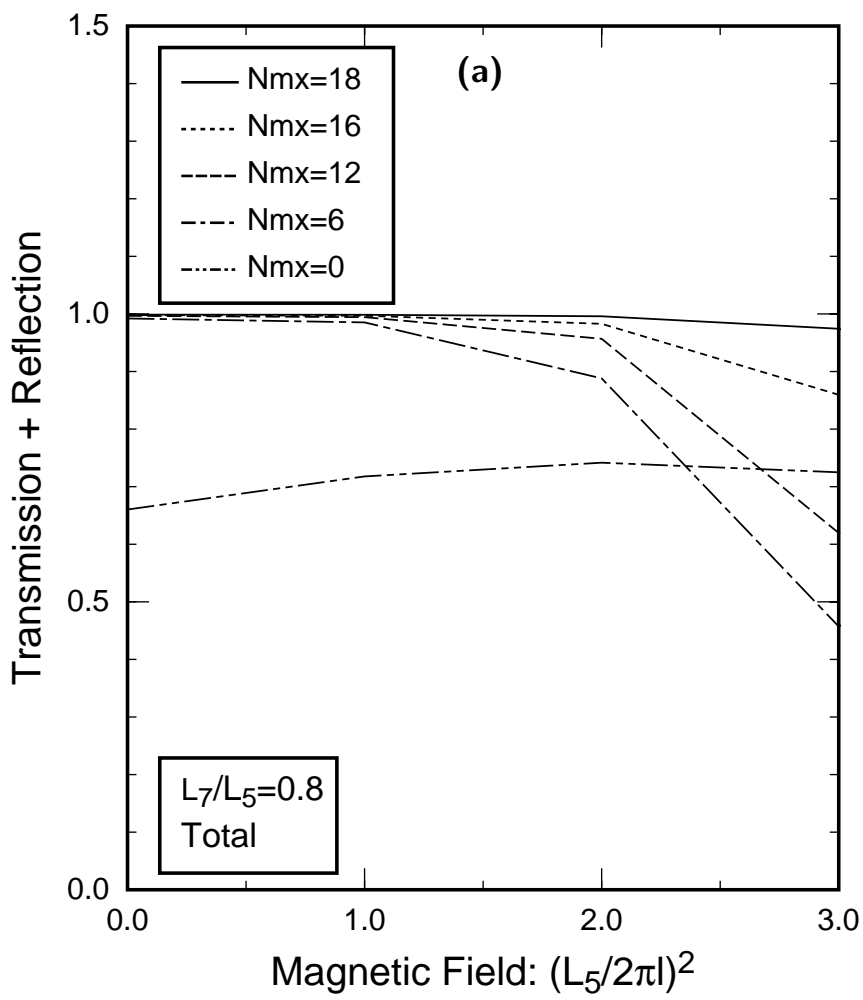

Fig. 9(a)

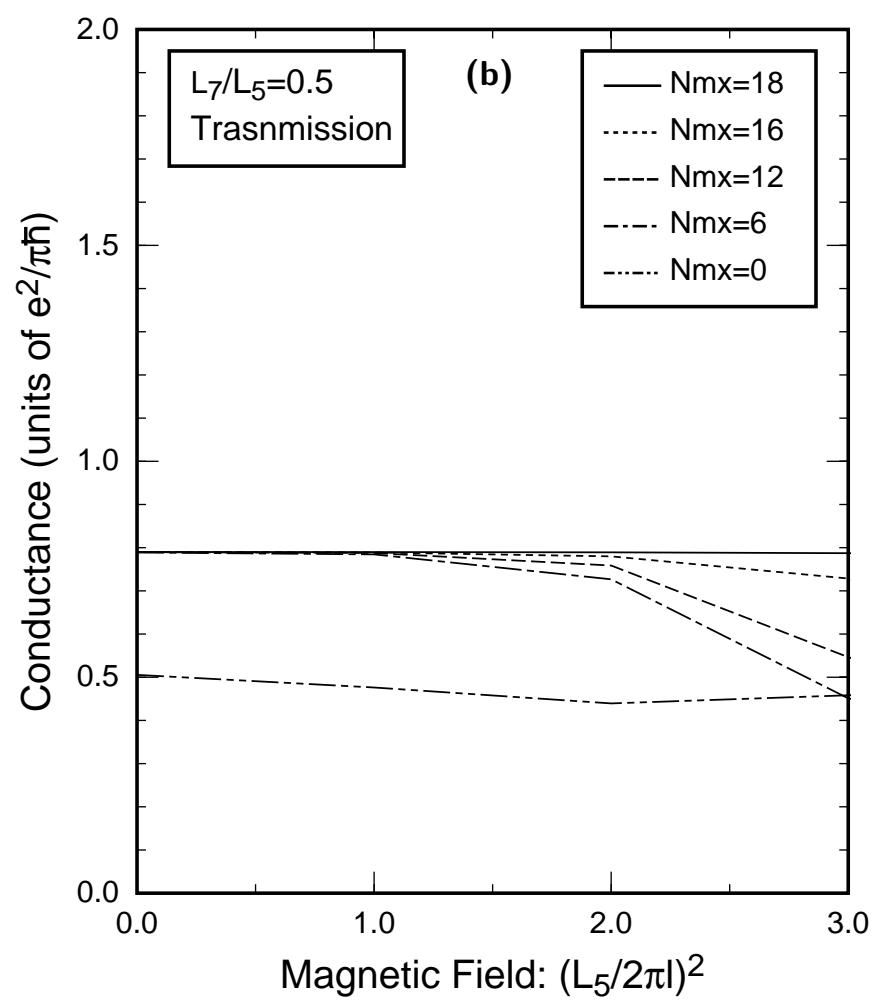

Fig. 8(b)

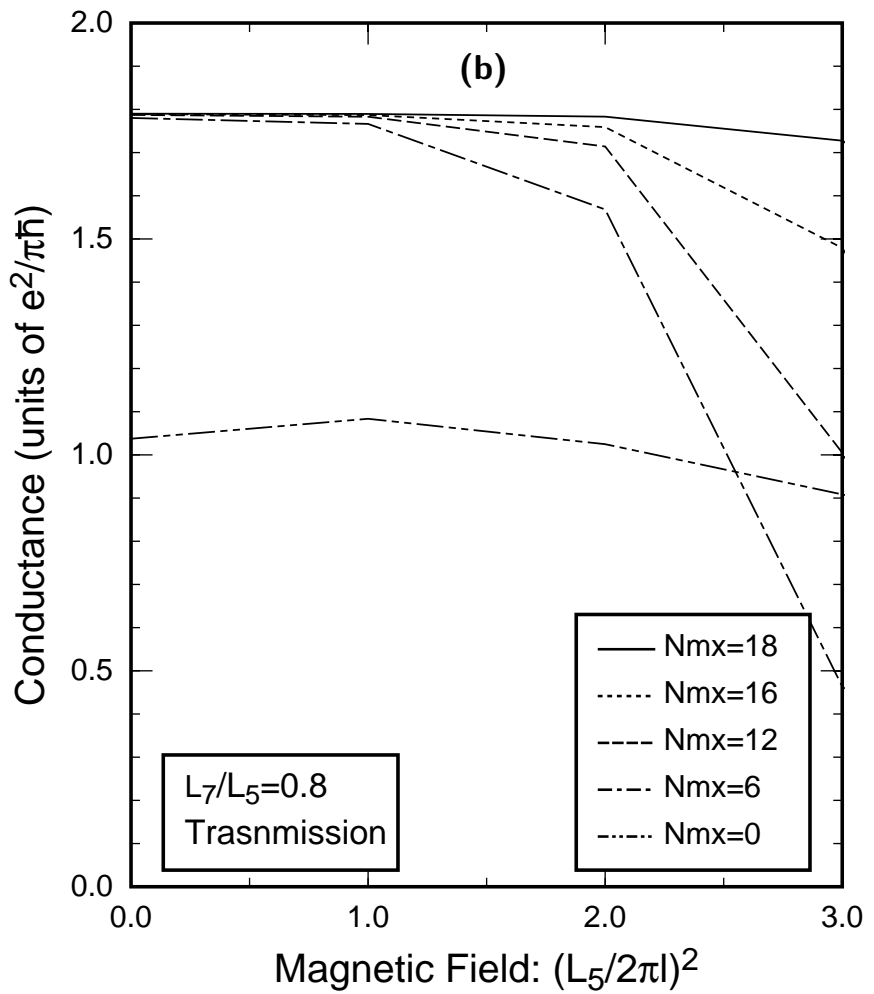

Fig. 9(b) 